\title{
Short wavelength ion temperature gradient mode and coupling with trapped electrons
}

\author{
J. Chowdhury, ${ }^{1}$ R. Ganesh, ${ }^{1, a)}$ J. Vaclavik, ${ }^{2}$ S. Brunner, ${ }^{2}$ L. Villard, ${ }^{2}$ and P. Angelino ${ }^{3}$ \\ ${ }^{1}$ Institute For Plasma Research, Bhat, Gandhinagar 382428, India \\ ${ }^{2}$ CRPP, Association EURATOM-Confédération Suisse, EPFL, 1015 Lausanne, Switzerland \\ ${ }^{3}$ CEA/DSM/DRFC, Association Euratom-CEA, Cadarache, 13108 St Paul-lez-Durance, France
}

(Received 21 July 2009; accepted 6 August 2009; published online 31 August 2009)

\begin{abstract}
The effect of trapped electrons on the ion temperature gradient (ITG) mode in a regime where its wavelength is shorter than the conventional ITG mode $\left(k_{\perp} \rho_{L i} \leq 1\right)$ has been studied. Such a mode propagates in the ion diamagnetic direction with a typical scale length $k_{\perp} \rho_{L i} \gg 1$ and is termed as the short wavelength ITG (SWITG) mode. The effect of the trapped electrons on this SWITG mode is investigated, for the first time, using a global and local linear gyrokinetic model. The trapped electrons are observed to destabilize the mode strongly. Comparison of the various parameter scans for the SWITG mode with and without the trapped electrons is presented. One important result obtained is that, while in the absence of the trapped electrons the mode was found to subside with increasing value of $\epsilon_{n}=L_{n} / R$ exhibiting the character of a slablike mode, the presence of the trapped electrons has been observed to enhance the $\epsilon_{n}=L_{n} / R$ window of the existence of the SWITG mode making the mode more toroidal like. (C) 2009 American Institute of Physics.
\end{abstract}

[DOI: $10.1063 / 1.3212890]$

\section{INTRODUCTION}

Controlling anomalous transport to achieve better confinement of plasma is one of the important issues in the present day fusion research. Low frequency (compared to the ion gyromotion) and fine scale length (compared to the ion Larmor radius) instabilities are considered to be responsible for the deterioration of the confinement, leading to the anomalous transport of particles and energy. These modes draw their free energy from the density and temperature inhomogeneities prevailing in a confined plasma. While electron transport exhibits multiscale feature ranging from the electron Larmor radius $\left(k_{\perp} \rho_{L i} \gg 1\right)$ of electron temperature gradient $(\mathrm{ETG})^{1-5}$ mode to the ion Larmor radius $\left(k_{\perp} \rho_{L i}\right.$ $\leq 1$ ) of the trapped electron mode, ${ }^{6-9}$ ion transport driven by the ion temperature gradient (ITG) ${ }^{10-12}$ mode, on the other hand, is known to be unstable at one scale length on the order of the ion Larmor radius $\left(k_{\perp} \rho_{L i} \leq 1\right)$.

However, of late, a new mode, with mode frequency in the direction of the ion diamagnetic drift frequency, on the intermediate scale between ITG and ETG modes with $k_{\perp} \rho_{L i}>1$ has been identified. ${ }^{13}$ This mode is found to be driven by the temperature gradient of the ions in the presence of the Landau resonance/inverse resonance in a slab geometry and by the toroidal drift resonance in a toroidal geometry, in combination with the nonmonotonic behavior of the mode frequency with respect to the perpendicular wave number. Because of its occurrence in the short wavelength limit and due to the nonadiabaticity of ions, the mode is named as short wavelength ITG (SWITG) mode to distinguish it from the conventional ITG mode at longer wavelength. It is generally speculated that, in the limit $\left(k_{\perp} \rho_{L i}\right)^{2} \gg 1$, there should be no mode intrinsic to the ion nonadiabaticity, since ion

\footnotetext{
${ }^{a)}$ Electronic mail: ganesh@ipr.res.in.
}

dynamics in this limit is expected to be adiabatic. However, if the scale length of the inhomogeneity is such that $\omega_{* i}$, the ion diamagnetic drift frequency becomes larger than the mode frequency $\omega$, there can be an instability related to the inhomogeneity in the ions even in this shorter limit. ${ }^{14}$

Initially, the mode was thought to be of hybrid type, ${ }^{14,15}$ requiring both $\eta_{i}$ and $\eta_{e}$ (ratio of the density to the temperature scale length of the ions and electrons, respectively) to be above a threshold. Later parametric study by Gao et al. ${ }^{16}$ demonstrated that the electron nonadiabaticity is not an essential ingredient for the mode to develop. Effect of the nonadiabatic electrons is only to enhance the growth rate of the mode. The theoretical study of this mode started with the work of Smolyakov et al. ${ }^{13}$ in a sheared slab and toroidal geometry using a local formulation. The work was then extended by Hirose et al. ${ }^{14}$ using a kinetic integral code based on ballooning formalism. This was followed by the study of the mode in the sheared slab ${ }^{15}$ and then in the toroidal geometry by Gao et al. ${ }^{16}$ Effects of shear flows on this mode have been studied in the sheared slab geometry and found to have strong stabilizing impact on the mode. ${ }^{17}$ However, it is expected that the toroidal SWITG mode will need higher rate of EXB flow shear for stabilization than the conventional toroidal ITG mode as the former has higher frequency. ${ }^{16}$ Dependence of the critical gradient on the various physical parameters such as temperature ratio, toroidicity, magnetic shear, and safety factor has been studied for this mode ${ }^{18}$ It is to be noted that such a double hump behavior was pointed out a long way back by $\mathrm{Pu}$ et al. ${ }^{19}$ while studying the ion mixing mode.

The main conclusions, from the past works are the following.

(1) In the slab limit (small toroidicity $\epsilon_{n}=L_{n} / R$ ), a strong temperature gradient driven mode exists in the regime 
$\left(k_{\perp} \rho_{L i}\right)^{2} \gg 1$. The instability requires both $\eta_{i}$ and $\eta_{e}$ to be above a critical value. ${ }^{14,15}$ But later study ${ }^{16}$ reveals that it is inherently an ion mode and exists even if the electrons are adiabatic. The same is observed in the work of Smolyakov et al. ${ }^{13}$

(2) Toroidicity has strong stabilizing effect on the mode. ${ }^{14}$ Stabilization occurs at $\epsilon_{n} \geq 0.15$.

(3) The instability is driven by magnetic shear and the growth rate is approximately proportional to $\sqrt{|s|},{ }^{14}$ where $s$ stands for the shear. But a broader parameter scan $^{16}$ finds that the growth rate initially increases and then starts decreasing with shear.

(4) Similar to the conventional ITG, it is also stabilized by a modest $\alpha$, the ballooning parameter.

(5) Nonadiabatic circulating electron dynamics provide destabilization.

(6) EXB flow shear has strong stabilizing effect on the mode.

In all the aforementioned studies, the trapped electrons were ignored. However, in a toroidal device, the trapped electrons are inevitable, and as demonstrated in the present work, can play a paramount role in defining the stability properties of the mode. In the limit $\omega_{b e}>\omega$, where $\omega_{b e}$ is the bounce frequency of the trapped electrons, the trapping of the electrons prevents the thermalization along the magnetic field line and the wave field appears stationary during a bounce period. The trapped electrons, therefore, can alter the stability properties of the mode significantly.

The other lacuna of the earlier studies is that they were done either using a local kinetic theory or at the best kinetic theory based on ballooning formalism in the slab as well as toroidal geometry. A ballooning formalism is essentially a one dimensional model in $\theta_{b}$, the ballooning angle. Therefore, the estimation of the characteristic radial scale length of the mode is not possible unless one uses the higher order ballooning theory. Thus, to understand the two dimensional mode structure in the presence of the trapped electrons, a global model becomes necessary. Also, an estimation of the radial scale length of the mode is not only required for completeness but also help estimate the probable transport induced by the mode. In fact, for the first time, the two dimensional SWITG mode structure will be shown in the present work. For the parameters chosen, the SWITG mode is found to be quite global.

Thus, the purpose of the present work is twofold: first, to incorporate the trapped electrons to the SWITG mode and, second, to use a global linear electrostatic gyrokinetic model, which enables one to evaluate the two dimensional mode structure of the SWITG mode.

The inclusion of the trapped electrons has drastic effect on the growth rate as well as the real frequency of the SWITG mode, in contrast to the earlier speculation that the trapped electrons may not be significant for the mode. The trapped electrons enhance the growth rate of the mode substantially because of the nonideal effects such as the magnetic drift resonance and reduction in the adiabatic fraction of the electrons. The trapped electrons enhance the real frequency which may lead to weaker Landau damping of the wave by the ions. This perhaps is another reason of the mode getting unstable in the presence of the trapped electrons. The parameter regime of the existence of the mode, consequently, gets widened introducing new domain of instability. Also, the mode structure of the so-called SWITG mode has been observed to be quite global, even though it exists at short wavelength compared to the ion Larmor radius. The mode structure spans over a substantial fraction of the tokamak poloidal cross section.

In this work, we use the electrostatic version of the widely used ${ }^{20-27}$ fully electromagnetic code EM-GLOGYSTO based on a global linear gyrokinetic model, treating all the species gyrokinetically as applicable to the large aspect ratio tokamak. The model uses finite Larmor stabilization (FLR) effect to all orders, all the kinetic resonances, poloidal and radial coupling due to the particle drifts across the magnetic flux surfaces, the trapped electrons and fully nonadiabatic ions, true ion to electron mass ratio, and profile variation. The perturbed $B_{\|}$and $B_{\perp}$ components of the magnetic field, Shafranov shift, and equilibrium flow have been dropped. A local version of this gyrokinetic formulation is also used for the purpose of comparison.

The subsequent parts are arranged as follows. Section II presents the basic set of gyrokinetic equations for the global as well as the local formulation; Sec. III addresses the results of our numerical study using both the local and the global gyrokinetic formulation. The dispersion diagrams of the SWITG mode with and without the trapped electrons followed by a comparative study of the parameter dependence of the mode for the two cases will be discussed one by one. Finally Sec. IV presents the conclusion from the results obtained.

\section{MODEL EQUATIONS}

\section{A. Global formulation}

In real space $\mathbf{r}$, for the species $j$, the perturbed density can be expressed as

$$
\begin{aligned}
\tilde{n}_{j}(\mathbf{r} ; \omega)= & -\left(\frac{q_{j} N}{T_{j}}\right)\left[\widetilde{\varphi}+\int d \mathbf{k} \exp (\iota \mathbf{k} \cdot \mathbf{r})\right. \\
& \left.\times \int d \mathbf{v} \frac{f_{M j}}{N}\left(\omega-\omega_{j}^{*}\right)\left(\mathcal{U}_{j}, \iota \mathcal{T}_{j}\right) \tilde{\varphi}(\mathbf{k} ;) J_{0}^{2}\left(x_{L j}\right)\right],
\end{aligned}
$$

where the first term on the right hand side corresponds to the adiabatic response while the second term represents the nonadiabatic response of the particles to a perturbation with all its kinetic effects. Here, $q_{j}$ and $T_{j}$ stand, respectively, for the charge and temperature of the species $j, N$ is the equilibrium density, $\omega_{j}^{*}=\omega_{n j}\left[1+\left(\eta_{j} / 2\right)\left(\left(v_{\|}^{2} / v_{\text {thj }}^{2}\right)-3\right)+\eta_{j} v_{\perp}^{2} / 2 v_{\text {th } j}^{2}\right]$ with $\omega_{n j}=\left(T_{j} \nabla_{n} \ln N k_{\theta}\right) /\left(q_{j} B\right)$ is the diamagnetic drift frequency; $\eta_{j}=\left(d \ln T_{j}\right) /(d \ln N)$ is the ratio of the density to temperature gradient scale lengths, $v_{\|}$and $v_{\perp}$ represent, respectively, the parallel and the perpendicular velocity, $v_{\text {th } j}$ is the thermal velocity of the species $j . J_{0}\left(x_{L j}\right)$ is the Bessel function of argument $x_{L j}=k_{\perp} \rho_{L j}$, accounting for the finite 
Larmor radius effect to all orders. We consider a local Maxwellian for each species of mass $m_{j}$ as

$$
f_{M j}(\xi, \psi)=\frac{N(\psi)}{\left[\frac{2 \pi T_{j}(\psi)}{m_{j}}\right]^{3 / 2}} \exp \left[-\frac{\xi}{T_{j}(\psi) / m_{j}}\right],
$$

where $\xi=v^{2} / 2$. Also in Eq. (1) $\mathcal{U}_{j}$ represents the guiding center propagator for the passing particles of the types $j$ $=i, e$ and $\mathcal{T}_{j}$ represents the guiding center propagator for the trapped particles of the types $j=i, e$ both describing the equilibrium guiding center motion. For details of the propagators for both passing and trapped particles the reader is referred to the Refs. 20, 24, and 25. Introducing the quasineutrality condition, one can close the set of equations in $\widetilde{n}_{j}$ as

$$
\sum_{j} \tilde{n}_{j}(\mathbf{r} ; \omega)=0 .
$$

One would, thus, finally end up with a generalized eigenvalue problem where $\omega$ and $\widetilde{\varphi}$, respectively, are the eigenvalue and the eigenvector. This can be then conveniently solved in the Fourier space by Fourier decomposing the potential in Eq. (2) first and then taking the Fourier transform, to eventually obtain a convolution matrix in the Fourier space. With the single charged passing ions, electrons along with the trapped electrons we have

$$
\sum_{\mathbf{k}^{\prime}} \sum_{j=i, e} \hat{\mathcal{M}}_{\mathbf{k}, \mathbf{k}^{\prime}}^{j} \widetilde{\varphi}_{\mathbf{k}^{\prime}}+\sum_{\mathbf{k}^{\prime}} \sum_{j=t r-e} \hat{\mathcal{M}}_{\mathbf{k}, \mathbf{k}^{\prime}}^{j} \widetilde{\varphi}_{\mathbf{k}^{\prime}}=0 .
$$

The axisymmetry of the system considered here enables one in the linear analysis to fix the toroidal mode number $n$, so that the notation $\mathbf{k}=(\kappa, m)$ for the wave vector defines the radial wave number $\kappa$ and the poloidal wave number $m$. Thus, $\mathbf{k}=(\kappa, m)$ and $\mathbf{k}^{\prime}=\left(\kappa^{\prime}, m^{\prime}\right)$. Note that we have considered three species here: nonadiabatic passing ions $(i)$, adiabatic passing electrons $(e)$, and trapped electrons $(\operatorname{tr}-e)$.

\section{B. Diagnostics: Eigenmode-averaged quantities}

Simple diagnostics for various physical quantities are computed as averages over the eigenmode. For example, mode-averaged $k_{\theta}^{2}$ is computed as

$$
\left\langle k_{\theta}^{2}\right\rangle=\frac{\int d \rho \sum_{m}\left|\frac{m}{\rho} \varphi_{(k, m)}\right|^{2}}{\int d \rho \sum_{m}\left|\varphi_{(k, m)}\right|^{2}},
$$

where quantities with suffix $(k, m)$ imply Fourier weights of corresponding perturbations.

\section{Local formulation}

Integrating the following local gyrokinetic equation, for which $k_{\perp} \simeq k_{\theta}$ and $k_{\|}=$constant, to get the perturbed density:

$$
f_{j}=-\frac{q_{j} F_{M j}}{T_{j}} \widetilde{\phi}+\frac{q_{j} F_{M i}}{T_{j}}\left(\omega-\omega_{* j}\right)\left(i \mathcal{U}_{j}, i \mathcal{T}_{j}\right) J_{o}^{2}\left(k_{\perp} \rho_{j}\right) \widetilde{\phi}
$$

one can write $\tilde{n}_{j}$ as
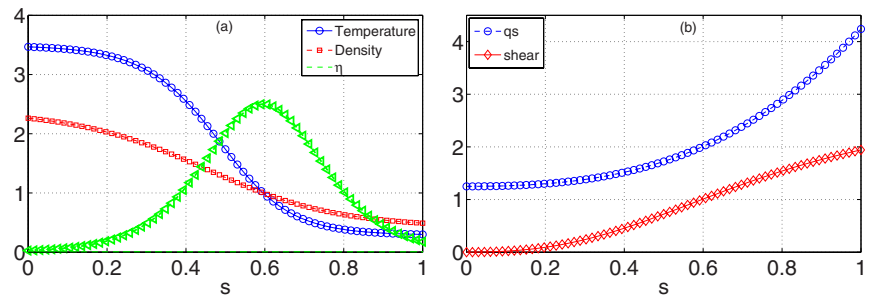

FIG. 1. (Color online) Equilibrium profiles to study the global SWITG mode (for parameters in Table I): (a) normalized density (square), temperature (circle), $\eta_{i, e}$ (triangle), (b) safety factor $q$ (circle), and magnetic shear $\hat{s}$ (diamond) profiles as functions of normalized radius $s=r / a$. Note that $\eta$ peaks at $s=\rho / a=s_{0}=0.6$ and is equal to 2.5. Also $q\left(s_{0}=0.6\right)=2.0, \hat{s}\left(s_{0}\right.$ $=0.6)=1.0, \epsilon_{n}\left(s_{0}=0.6\right)=0.1$, and $\tau\left(s_{0}=0.6\right)=1.0$.

$$
\begin{aligned}
\widetilde{n}_{j}(k)= & -\frac{q_{j} N_{j}}{T_{j}}\left[1-\frac{1}{\sqrt{2 \pi} v_{\mathrm{thj}}^{3}} \int d v_{\perp} d v_{\|} v_{\perp} e^{-v^{2} / 2 v_{\mathrm{thj}}^{2}(\omega}\right. \\
& \left.\left.-\omega_{* j}\right)\left(i \mathcal{U}_{j}, i \mathcal{T}_{j}\right) J_{0}^{2}\left(k_{\perp} \rho_{L j}\right)\right] \widetilde{\phi},
\end{aligned}
$$

where the propagator for the untrapped particles is given by $i \mathcal{U}_{j}=1 /\left(\omega-k_{\|} v_{\|}-\omega_{d j}\right)$, while that of the trapped particles is given by $i \mathcal{T}_{j}=1 /\left(\omega-\omega_{d j}\right)$. Using the quasineutrality condition and considering the passing electrons to be adiabatic and adding the trapped electrons, one would finally get

$$
1+\tau-\sqrt{(2 \epsilon)} I_{00}^{\mathrm{tr}-e}-\tau I_{00}^{i}=0 .
$$

The trapped electron integral $I_{00}^{\mathrm{tr}-e}$ is weighted by the trapped fraction $\sqrt{2 \epsilon}, \epsilon=r / a$, and $\tau=T_{e} / T_{i}$. Here we put

$$
\begin{aligned}
\widetilde{I}_{l, p}^{j}= & \frac{1}{\sqrt{2 \pi} v_{\text {th } j}^{3}} \int d v_{\perp} d v_{\|} v_{\perp} e^{-v^{2} / 2 v_{\text {th }}^{2}\left(\omega-\omega_{* j}\right)\left(i \mathcal{U}_{j}, i \mathcal{T}_{j}\right)} \\
& \times\left(\frac{v_{\|}}{v_{\text {th }}}\right)^{l}\left(\frac{v_{\perp}}{v_{\text {th } j}}\right)^{p} J_{0}^{2}\left(k_{\perp} \rho_{L j}\right) .
\end{aligned}
$$

\section{RESULTS AND DISCUSSION}

In the present section, we will delineate the results from the global and the local gyrokinetic formulation and compare the cases of SWITG without the trapped electrons and with the trapped electrons. It is to be noted that the frequencies are normalized with $v_{\text {thi }} / a$ throughout the paper. Let us consider the following profiles and parameters.

\begin{tabular}{lc}
\begin{tabular}{lc} 
Parameters & Equilibrium profiles \\
\hline$B$-field: $B_{0}=1.0$ Tesla & N-profile and T-profile \\
Temperature: $T_{0}=T\left(s_{0}\right)=7.5 \mathrm{keV}$ & $N(s) / N_{0}=\exp \left[-\frac{a \delta s_{n}}{L_{n}} \tanh \left(\frac{s-s_{0}}{\delta s_{n}}\right)\right]$ \\
Major radius: $R=2.0 \mathrm{~m}$ & $T_{i, e}(s) / T_{0}=\exp \left[-\frac{a \delta s_{T}}{L_{T 0}} \tanh \left(\frac{s-s_{0}}{\delta s_{T}}\right)\right]$ \\
Minor radius: $a=0.5 \mathrm{~m}$ & $\delta s_{n}=0.35, \delta s_{T}=0.2$ at $s=s_{0}$ \\
radius: $s=\rho / a, 0.01<s<1.0, s_{0}=0.6$ & $q(s)=1.25+0.67 \mathrm{~s}^{2}+2.38 \mathrm{~s}^{3}-0.06 \mathrm{~s}^{4}$ \\
$L_{n 0}=0.2 \mathrm{~m}, L_{T 0}=0.08 \mathrm{~m} \rightarrow \eta_{i, e}\left(s_{0}\right)=2.5$ & such that $q\left(s=s_{0}\right)=2.0 ;$ \\
$\tau(s)=T_{e}(s) / T_{i}(s)=1, \epsilon_{n}=L_{n 0} / R=0.1$ & Shear $\hat{s}$ is positive and $\hat{s}=1$ at $s=s_{0}$
\end{tabular} \\
\hline
\end{tabular}

The equilibrium profiles corresponding to these parameters are shown in Fig. 1. The chosen parameters lead to the value of $\rho^{*} \equiv \rho_{L i}\left(s=s_{0}\right) / a \simeq 0.0175$. 


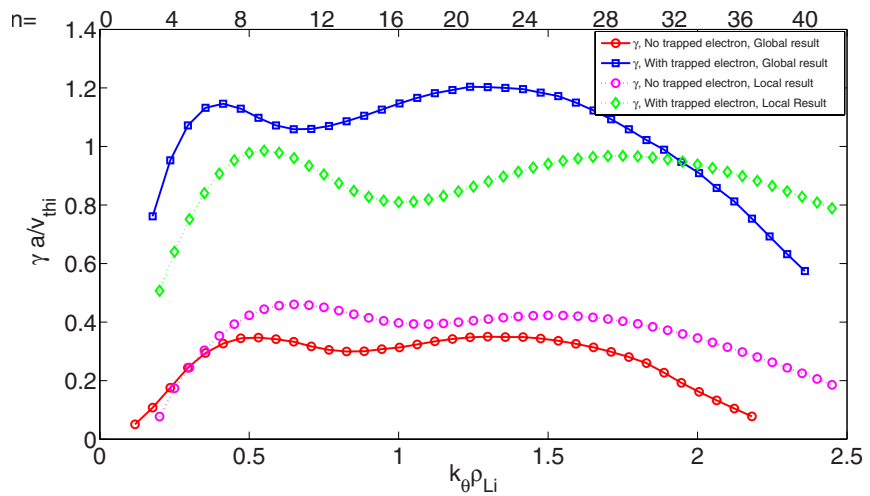

FIG. 2. (Color online) The normalized growth rate $\tilde{\gamma}$ of the ITG (first peak) and of the SWITG (second peak) mode with (solid line+square) and without (solid line+circle) the trapped electrons from the global as well as the local formulation (dotted line+diamond for the case with the trapped electrons and dotted line+circle for the case without the trapped electrons) $\eta_{e, i}\left(s_{0}\right)$ $=2.5, q\left(s_{0}\right)=2.0, \hat{s}\left(s_{0}\right)=1.0, \tau=1.0$, and $\epsilon_{n}=0.1$. The upper axis shows the corresponding toroidal mode numbers.

\section{A. $\boldsymbol{k}_{\theta} \rho_{L i} \operatorname{scan}$}

Figure 2 shows the growth rates of the SWITG mode with respect to $k_{\theta} \rho_{L i}$ for the case (1) without the trapped electrons and (2) with the trapped electrons as obtained with the global as well as the local gyrokinetic model. The upper axis displays the corresponding toroidal mode numbers $n$. Let us first consider the curve with solid line and open circles. This presents the growth rate from the global model versus $k_{\theta} \rho_{L i}$ for the SWITG mode without the trapped electrons. The growth rate increases at lower $k_{\theta} \rho_{L i}$, peaks at $k_{\theta} \rho_{L i} \approx 0.5, n=9$, and then starts falling again, with a minimum at $k_{\theta} \rho_{L i} \approx 0.8, n=14$. After this point, the growth rate exhibits a similar trend as the first hump and peaks at $k_{\theta} \rho_{L i}$ $\approx 1.3, n=21$. The dotted line with open circles is the similar curve obtained from the local model without the trapped electrons. The peaks for both the conventional ITG and the SWITG modes are shifted toward higher $k_{\theta} \rho_{L i}$ in comparison with the global results. The first peak appears at $k_{\theta} \rho_{L i}$ $\approx 0.65$ and the second peak appears at $k_{\theta} \rho_{L i} \approx 1.5$ corresponding, respectively, to the ITG and the SWITG mode. The growth rates are slightly higher in the case of the local results than those obtained in the case of the global results. The real frequency as shown in Fig. 3 (solid line with open circle for the global model and dotted line with open circle for the local model), on the other hand, increases with $k_{\theta} \rho_{L i}$ up to the point $k_{\theta} \rho_{L i} \approx 0.8$ and $k_{\theta} \rho_{L i} \approx 1.3$, respectively, for the global and the local model. Beyond this point, the frequency starts to behave nonmonotonically with $k_{\theta} \rho_{L i}$. For both the cases, the first hump corresponds to the conventional ITG mode. In this region, the mode frequency being proportional to the $k_{\theta} \rho_{L i}$ increases almost linearly with $k_{\theta} \rho_{L i}$. The ITG mode then smoothly changes to the high $k$ SWITG mode. The nonmonotonic part can be considered as one of the characteristics of the SWITG mode. From Fig. 2, it is clear that the SWITG mode also suffers FLR stabilization like the conventional ITG; the mode growth rate increases initially then peaks at $k_{\theta} \rho_{L i} \approx 1.3\left(k_{\theta} \rho_{L i} \approx 1.5\right)$ for the global (local) mode and then starts falling.

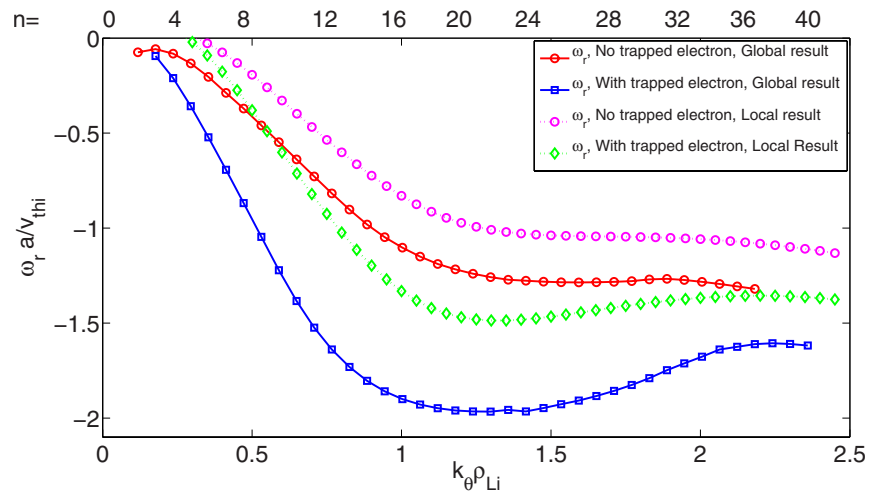

FIG. 3. (Color online) The normalized real frequency $\widetilde{\omega}_{r}$ of the ITG and of the SWITG mode with (solid line+square) and without (solid line+circle) the trapped electrons from the global as well as the local formulation (dotted line+diamond for the case with the trapped electrons and dotted line + circle for the case without the trapped electrons) $\eta_{e, i}\left(s_{0}\right)=2.5, q\left(s_{0}\right)=2.0$, $\hat{s}\left(s_{0}\right)=1.0, \tau=1.0$, and $\epsilon_{n}=0.1$. The upper axis shows the corresponding toroidal mode numbers.

Following the formulation of Gao et al., ${ }^{15,16}$ the nonmonotonic behavior of the real frequency and the double humped growth rate can be explained qualitatively using the local gyrokinetic model for which $k_{\perp} \simeq k_{\theta}$. Let us rewrite the perturbed distribution function $\tilde{f}_{i}$ for the ions as

$$
f_{i}=-\frac{q_{i} F_{M i}}{T_{i}} \widetilde{\phi}+\frac{q_{i} F_{M i}}{T_{i}}\left(\frac{\omega-\omega_{* i}}{\omega-\omega_{d i}-k_{\|} v_{\|}}\right) J_{o}^{2}\left(k_{\perp} \rho_{L i}\right) \widetilde{\phi}
$$

The first part corresponds to the adiabatic response while the second part corresponds to the nonadiabatic response of the ions. Integrating over velocity, to get the perturbed density for the ions in the limit $\omega_{n}>\omega>\left(\omega_{d i}+k_{\|} v_{\|}\right)$, one can write

$$
\widetilde{n}_{i}=-\frac{q_{i} n_{o}}{T_{i}} \widetilde{\phi}+\frac{q_{i}}{T_{i}} \widetilde{\phi} \frac{\omega_{n i}\left(\eta_{i} / 2-1\right)}{\omega} I_{0}\left(k_{\perp}^{2} \rho_{L i}^{2}\right) \exp \left(-k_{\perp}^{2} \rho_{L i}^{2}\right),
$$

where $I_{0}$ is the modified Bessel function of order zero. Since the SWITG mode can exist even with the adiabatic electrons and retains its basic characters, we for simplicity drop the nonadiabatic part of the electrons and consider them to be adiabatic, i.e., $\widetilde{n}_{e} / n_{o}=q_{e} \widetilde{\phi} / T_{e}$. The quasineutrality condition will then give

$$
\omega=\left(\frac{\tau}{\tau+1}\right)\left(\frac{\eta_{i}}{2}-1\right) \omega_{n i} I_{0}\left(k_{\perp}^{2} \rho_{L i}^{2}\right) \exp \left(-k_{\perp}^{2} \rho_{L i}^{2}\right),
$$

where $\omega_{n i}=-\left(v_{\text {thi }} / L_{n}\right)\left(k_{\perp} \rho_{L i}\right)$. Thus, it is clear from the expression that the mode frequency $\omega$ behaves as $\omega_{n i} I_{0}\left(k_{\perp}^{2} \rho_{L i}^{2}\right) \exp \left(-k_{\perp}^{2} \rho_{L i}^{2}\right)$ which for small $k_{\perp}^{2} \rho_{L i}^{2}$ scales as $k_{\perp} \rho_{L i}$ and for larger $k_{\perp}^{2} \rho_{L i}^{2}$ scales as almost a constant. It is because, from the property of the scaled modified Bessel function, one finds that $I_{0}\left(k_{\perp}^{2} \rho_{L i}^{2}\right) \exp \left(-k_{\perp}^{2} \rho_{L i}^{2}\right)$ $\rightarrow 1 / \sqrt{2 \pi\left(k_{\perp}^{2} \rho_{L i}^{2}\right)}=1 / \sqrt{2 \pi}\left(k_{\perp} \rho_{L i}\right)$ for large $k_{\perp}^{2} \rho_{L i}^{2}$ and $\omega_{n i}$ $\propto k_{\perp} \rho_{L i}$. This explains the nonmonotonic part of the real frequency. Regarding the growth rate, in the toroidal geometry, it is the toroidal magnetic drift term $\omega_{d i}$ of the ions, the resonance of which with the mode frequency gives rise to the double hump behavior. It is to be noted that $\omega_{d i}$ 
$\sim\left(L_{n} / R\right) \omega_{n i}$ and thus scales as $k_{\perp} \rho_{L i}$. Therefore, the ratio $\omega / \omega_{d i}$ at first increases for small $k_{\perp} \rho_{L i}$ and then decreases as the numerator saturates but the denominator still grows as $k_{\perp} \rho_{L i}$.

The FLR stabilization of the SWITG mode can be inferred from the nonadiabatic part of ion density response. At very high $k_{\perp} \rho_{L i}, \omega_{d i}$ surpasses $\omega$ and the nonadiabatic part of the perturbed ion density can be reduced to, for $\omega_{d i} \gg \omega$,

$$
\widetilde{n}_{i}^{n a}=\frac{q_{i}}{T_{i}} \widetilde{\phi} \frac{\omega_{n i}\left(\eta_{i} / 2-1\right)}{\omega_{d i}} I_{0}\left(k_{\perp}^{2} \rho_{L i}^{2}\right) \exp \left(-k_{\perp}^{2} \rho_{L i}^{2}\right),
$$

which for large $k_{\perp} \rho_{L i}$ will decrease according to

$$
\frac{\omega_{n i}}{\omega_{d i}} I_{0}\left(k_{\perp}^{2} \rho_{L i}^{2}\right) \exp \left(-k_{\perp}^{2} \rho_{L i}^{2}\right) \sim \frac{R}{L_{n}} I_{0}\left(k_{\perp}^{2} \rho_{L i}^{2}\right) \exp \left(-k_{\perp}^{2} \rho_{L i}^{2}\right)
$$

as $k_{\perp}^{2} \rho_{L i}^{2}$ increases.

Having elucidated the basic characters of the SWITG mode, let us now see what happens to the mode when trapped electrons are included. In Fig. 2 the solid line with squares represents the growth rates from the global model and the dotted line with diamonds represents the growth rates from the local model with trapped electrons present in both the cases. Similar curves in Fig. 3 represent the corresponding real frequencies. It is clear that for both the cases, the growth rate rises substantially in the presence of the trapped electrons. The mode frequencies also increase as compared to their counterparts with no trapped electrons. The global curve for growth rates peaks at $k_{\theta} \rho_{L i} \approx 0.4, n=7$, while the local curve peaks at $k_{\theta} \rho_{L i} \approx 0.55$ for the conventional ITG. For the SWITG mode, the growth rate peaks at $k_{\theta} \rho_{L i} \approx 1.3$, $n=21$, for the global result while it peaks at $k_{\theta} \rho_{L i} \approx 1.7$ for the local result. It is to be noted that the local growth rates stay below the global growth rates for most of the part of the $k$ spectrum. Beyond $k_{\theta} \rho_{L i} \approx 2.0$, the global growth rates fall faster than the local growth rates.

The strong rise in the growth rate of the SWITG mode in the presence of the trapped electrons can be explained, similar to the conventional ITG mode, as follows. In a toroidal plasma with ITG, a pressure perturbation in the outboard side creates hotter and colder regions locally. The magnetic drift velocity $v_{d}$ of the ions, which depends on the temperature, is therefore different in regions of different temperatures. This produces variations in the local concentration of the ion density giving rise to a potential perturbation and concurrently a poloidal electric field. The SWITG instability arises because of the radial EXB drift produced by this electric field in the presence of the applied magnetic field. When one considers electrons to be adiabatic, the moment charge separation is produced, these electrons move to the regions of charge separation and wipe out the space charge, thus denying the possibility of building up of EXB advection or reducing it. However, in a toroidal geometry, because of $1 / R$ dependence of the magnetic field, some electrons are "trapped" on the weaker magnetic field region and fail to

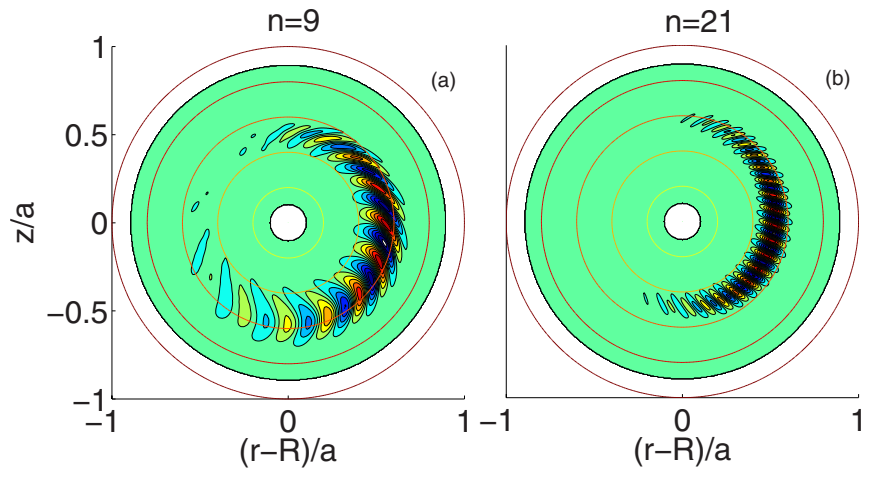

FIG. 4. (Color online) Two dimensional eigenmode structures of (a) the ITG mode at $k_{\theta} \rho_{L i} \approx 0.5, n=9$, (b) the SWITG mode at $k_{\theta} \rho_{L i} \approx 1.3, n=21$ without the trapped electrons both corresponding to the maximum growth rate. The mode structure of the SWITG mode is finer than the ITG mode but yet global enough.

behave adiabatically, in the sense that their motion is restricted to a limited region of the magnetic field lines. Trapped electrons, therefore, cannot respond adiabatically to reach the region of EXB advection and participate in canceling the charge separations, thereby allowing finite time for the mode to grow. The SWITG growth rate thus gets enhanced, when one considers the fraction of the trapped electrons.

The presence of the trapped electrons increases the real frequency and hence the phase velocity of the wave. This can perhaps make the wave off resonant with the ions and leads to weaker Landau damping of the wave by the ions with the concomitant enhancement in the growth rate. The SWITG growth rate, therefore, increases with the inclusion of the trapped electrons.

Figure 4 presents the mode structure of (a) the conventional ITG at $n=9$ and (b) the SWITG at $n=21$ both corresponding to the maximum growth rate without the trapped electrons. The eigenmode-averaged radial wave numbers for the two cases are $\left\langle k_{r} \rho_{L i}\right\rangle=0.687$ and $\left\langle k_{r} \rho_{L i}\right\rangle=0.702$, respectively. These figures show clearly that although the mode is termed as SWITG, its mode structure is quite global albeit lesser than the conventional ITG mode. The mode structure spans over a considerable fraction of the poloidal cross section of a tokamak. It corroborates the necessity of a global model to study the SWITG mode. Figure 5 then displays the mode structures, respectively, of the (a) conventional ITG mode with the trapped electrons at $n=7$ and (b) SWITG mode with the trapped electrons at $n=21$, both corresponding to the maximum growth rate of the mode. It is to be noted that the corresponding eigenmode-averaged radial wave numbers in these cases are $\left\langle k_{r} \rho_{L i}\right\rangle=0.489$ and $\left\langle k_{r} \rho_{L i}\right\rangle=1.132$, respectively. For clarity, we present a closeup view of the mode structures in Fig. 6 for the case without the trapped electrons with Fig. 6(a) for the conventional ITG mode and Fig. 6(b) for the SWITG mode and in Fig. 7 for the case with the trapped electrons with Fig. 7(a) for the conventional ITG mode and Fig. 7(b) for the SWITG mode.

Figure 8 portrays the poloidal Fourier components for the cases of (a) the ITG mode at $n=9$, (b) the SWITG mode at $n=21$ without the trapped electrons, (c) the ITG mode at 


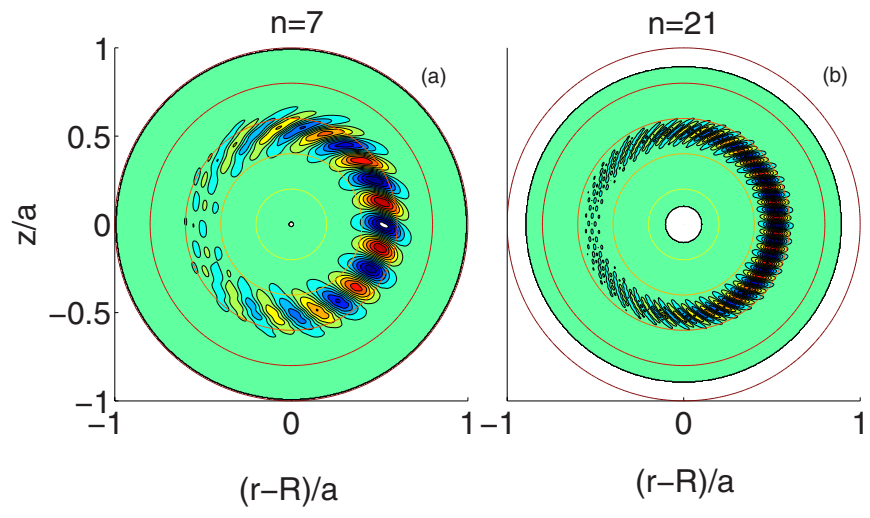

FIG. 5. (Color online) Two dimensional eigenmode structures of (a) the ITG mode at $k_{\theta} \rho_{L i} \approx 0.4, n=7$, (b) the SWITG mode at $k_{\theta} \rho_{L i} \approx 1.3, n=21$ with the trapped electrons, both for the maximum growth rates, respectively. The mode structure of the SWITG mode is finer than the ITG mode but still global enough.

$n=7$, and (d) the SWITG mode at $n=21$ with the trapped electrons. The strong poloidal coupling of the mode brought about by the cross field drift of the particles is quite apparent from these figures. Figure 9 shows the radial Fourier harmonics for the modes displayed in Figs. 4 and 5.

\section{B. $\epsilon_{n}$ scan}

The $L_{n} / R$ scan is performed varying $R$ but keeping $R q$, $n / R, a$, and $L_{n}$ constant. Figure 10 presents the growth rates for the two cases: (a) without the trapped electrons (circle) and (b) with the trapped electrons (square) from the global gyrokinetic formulation. Local results are not shown here. It is clear that the SWITG mode subsides more rapidly with $\epsilon_{n}$ and vanishes at around $\epsilon_{n} \sim 0.17$. It is argued, therefore, in the earlier literature that the SWITG mode is preferentially a slab mode which decays with increasing toroidicity. The decrease in the growth rate with $L_{n} / R$ can again be attributed to the reduction in the nonadiabatic fraction of the ion's perturbed density response with $L_{n} / R$, as it scales as inverse of $L_{n} / R$ as apparent from Eq. (10). Inclusion of the trapped electrons, however, widen the $L_{n} / R$ window. The mode sustains to a higher value of $L_{n} / R$. Thus, one concludes that the trapped electrons have deleterious effect on the SWITG mode enhancing not only its growth rate but also widening its parameter regime of existence. The fact is that, with in-
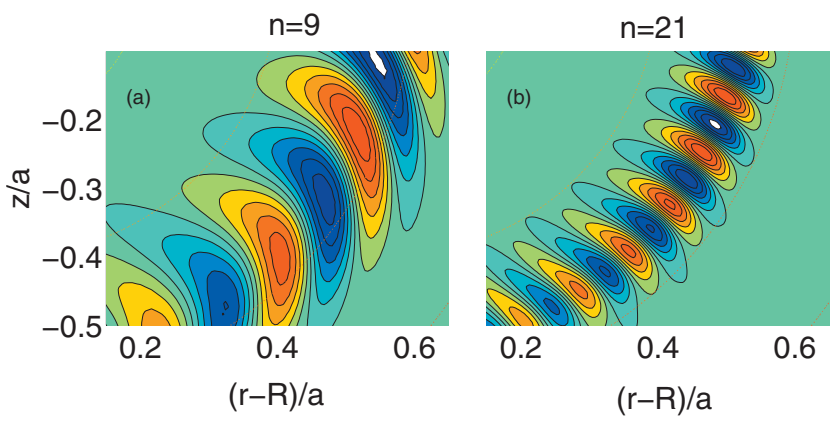

FIG. 6. (Color) A closeup view of the eigenmode structures of (a) the ITG mode at $k_{\theta} \rho_{L i} \approx 0.5, n=9$, (b) the SWITG mode at $k_{\theta} \rho_{L i} \approx 1.3, n=21$ without the trapped electrons shown in Fig. 4.

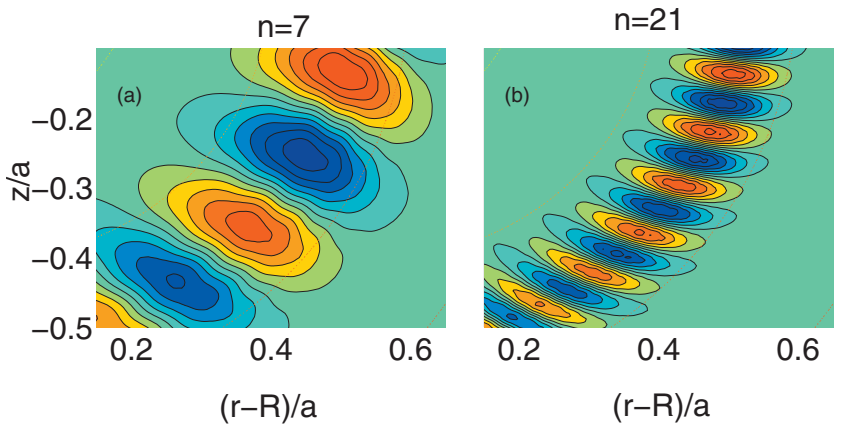

FIG. 7. (Color) A closeup of the two dimensional eigenmode structures of (a) the ITG mode at $k_{\theta} \rho_{L i} \approx 0.4, n=7$, (b) the SWITG mode at $k_{\theta} \rho_{L i} \approx 1.3$, $n=21$ with the trapped electrons shown in Fig. 5 .
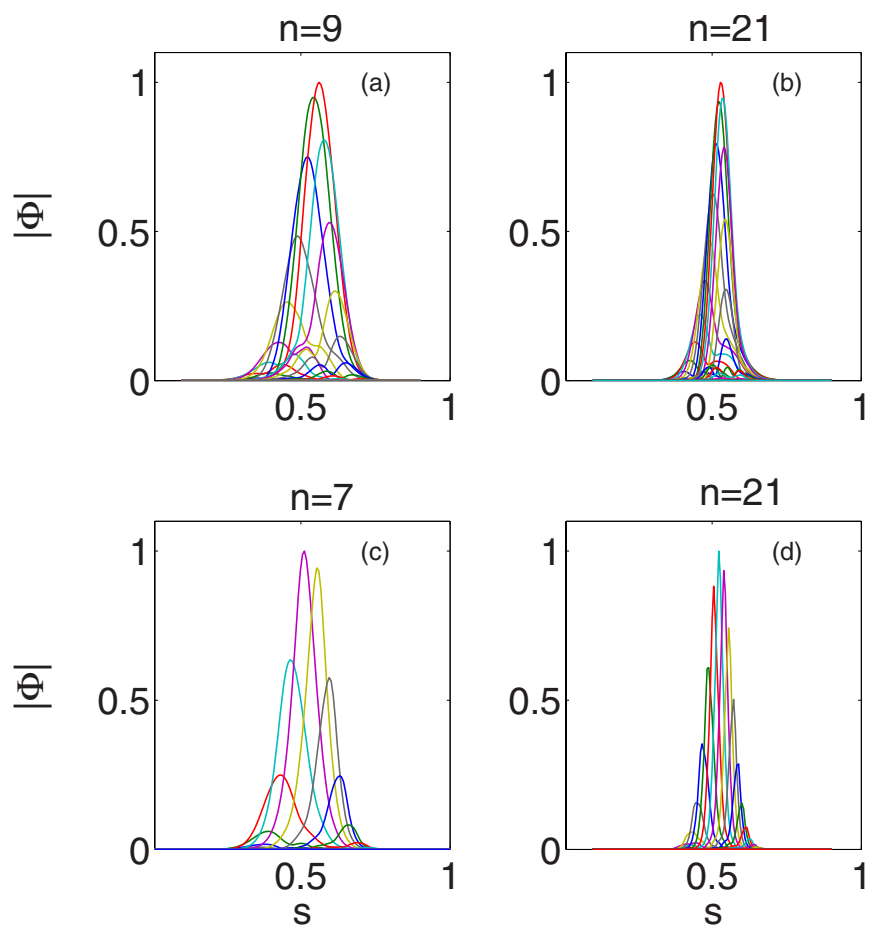

FIG. 8. (Color online) Poloidal Fourier components for electrostatic modes shown in Figs. 4 and 5, (a) the ITG mode at $k_{\theta} \rho_{L i} \approx 0.5, n=9$, (b) the SWITG mode at $k_{\theta} \rho_{L i} \approx 1.3, n=21$, both without the trapped electrons, (c) the ITG mode at $k_{\theta} \rho_{L i} \approx 0.4, n=7$, and (d) the SWITG mode at $k_{\theta} \rho_{L i} \approx 1.3$, $n=21$, both with the trapped electrons.
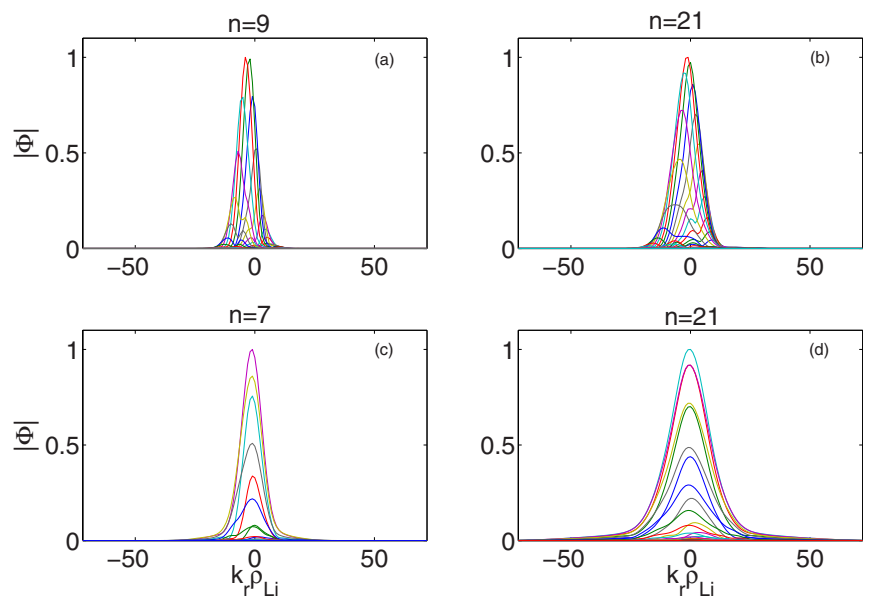

FIG. 9. (Color online) Radial Fourier components for electrostatic modes shown in Figs. 4 and 5 , (a) the ITG mode at $k_{\theta} \rho_{L i} \approx 0.5, n=9$, (b) the SWITG mode at $k_{\theta} \rho_{L i} \approx 1.3, n=21$, both without the trapped electrons, (c) the ITG mode at $k_{\theta} \rho_{L i} \approx 0.4, n=7$, (d) the SWITG mode at $k_{\theta} \rho_{L i} \approx 1.3, n$ $=21$, both with the trapped electrons. 


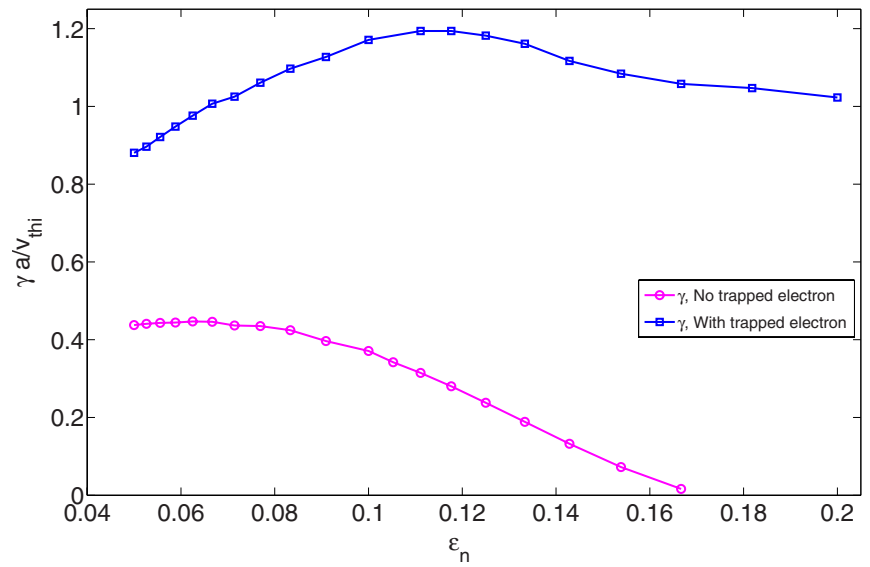

FIG. 10. (Color online) Normalized growth rates $\tilde{\gamma}$ vs $\epsilon_{n}$ scan for the SWITG mode at $k_{\theta} \rho_{L i} \approx 1.3$ with (solid line+square) and without (solid line+circles) the trapped electrons (from the global gyrokinetic model). $\eta_{e, i}\left(s_{0}\right)=2.5, q\left(s_{0}\right)=2.0, \hat{s}\left(s_{0}\right)=1.0, \tau=1.0, L_{n}=0.2$, and $a=0.5$.

creasing toroidicity the fraction of the trapped particles which is proportional to $\sqrt{r / R}$ also increases. Therefore, in contrast to the previous case of the SWITG where toroidicity has strong stabilizing effect, making the mode vanish beyond $L_{n} / R \sim 0.15$, the SWITG in the presence of the trapped electrons can exist above this limit, as the stabilizing effect of the toroidicity is compensated by the destabilizing effect of the trapped electrons. In Fig. 11, the corresponding real frequencies are shown. Solid line with circles presents the case without the trapped electrons and solid line with squares presents the case with the trapped electrons. While, with toroidicity the growth rates decay, real frequencies on the other hand increase with the toroidicity. Thus, although earlier SWITG was thought to be stable beyond some specific value of $\epsilon_{n}$, the trapped electrons can make it unstable for a general set of parameters. Therefore, the theories for experimentally observed anomalous transport, explained with the conventional ITG mode coupled with trapped electrons and the trapped electron mode as the plausible candidates, should be revisited. It is perhaps worth noting at this point that experimen-

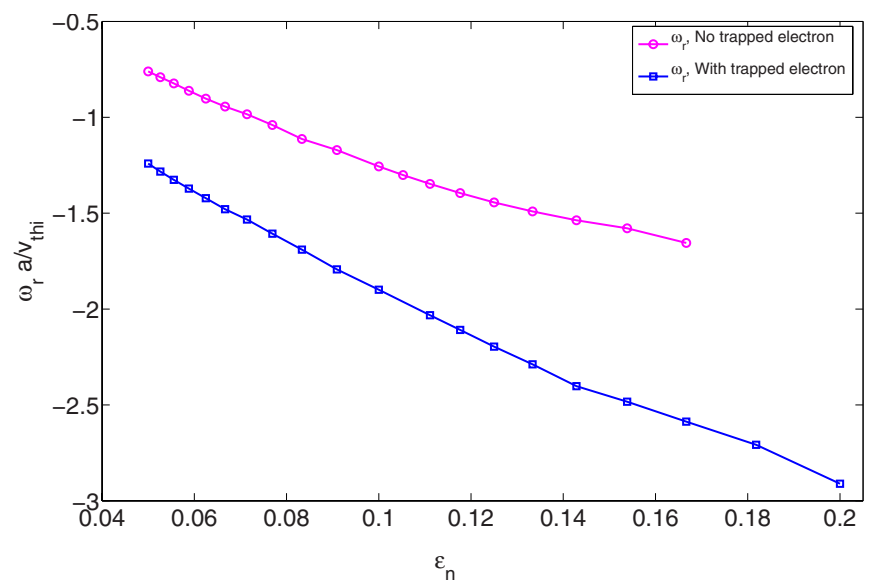

FIG. 11. (Color online) Normalized real frequency $\widetilde{\omega}_{r}$ vs $\epsilon_{n}$ scan for the SWITG mode at $k_{\theta} \rho_{L i} \approx 1.3$ with (solid line+square) and without (solid line + circles) the trapped electrons (from the global gyrokinetic model). $\eta_{e, i}\left(s_{0}\right)=2.5, q\left(s_{0}\right)=2.0, \hat{s}\left(s_{0}\right)=1.0, \tau=1.0, L_{n}=0.2$, and $a=0.5$.

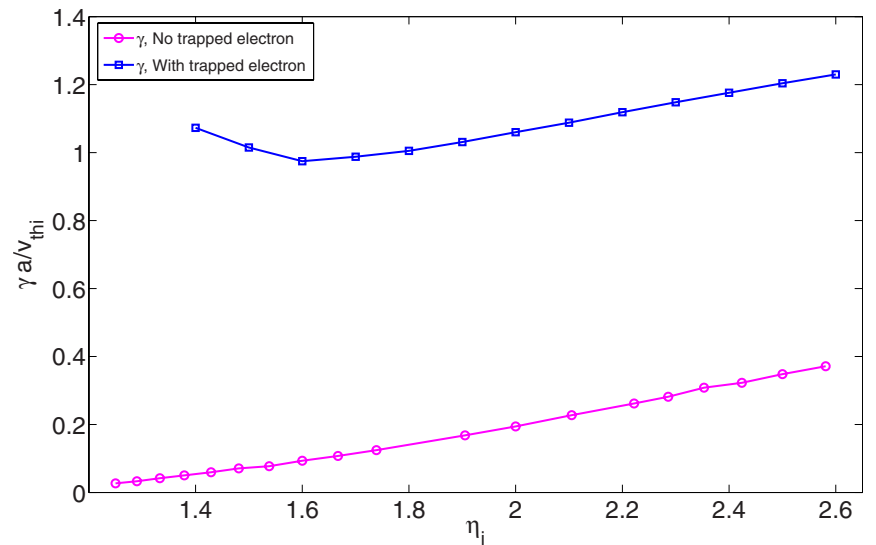

FIG. 12. (Color online) Normalized growth rates $\tilde{\gamma}$ vs $\eta_{i}$ scan for the SWITG mode at $k_{\theta} \rho_{L i} \approx 1.3$ with (solid line+square) and without (solid line+circles) the trapped electrons (from the global gyrokinetic model). $\eta_{e}\left(s_{0}\right)=2.5, q\left(s_{0}\right)=2.0, \hat{s}\left(s_{0}\right)=1.0, \tau=1.0$, and $\epsilon_{n}=0.1$.

tally Wong et $a l^{28}$ reported the observation of such a short wavelength fluctuation in the context of electron transport. The mode has frequency lower than the ion diamagnetic drift frequency and propagates in the ion diamagnetic direction as the SWITG mode studied here. However, $k_{\perp} \rho_{L i}$ measured is higher $(\sim 5)$ than the one found in our simulation.

\section{C. $\eta_{i}$ scan}

To demonstrate the temperature gradient dependence of the SWITG mode, an $\eta_{i}$ scan for the maximum growth rates is shown in Fig. 12 without (circles) and with (squares) the trapped electrons using the global gyrokinetic model. It is clear from the figure that the SWITG mode without the trapped electrons is inherently an ion mode, requiring no finite $\eta_{e}$ and solely depends on the temperature gradient of the ions. The dependence of the mode growth rate on $\eta_{i}$ is quite similar to the conventional ITG which decays with decreased $\eta_{i}$. The SWITG mode decays as one reduces $\eta_{i}$ and vanishes completely around $\eta_{i c} \sim 1.2$. Inclusion of the trapped electrons, on the other hand, restricts the mode to vanish, rather the mode transforms from the ITG driven mode to the trapped electrons driven mode. When the ion drive is reduced by reducing $\eta_{i}$, the growth rate decreases, but since the mode now includes the trapped electrons with finite $\eta_{e}$, mode inherent to the trapped electrons takes over the ion gradient driven mode. Thus, the SWITG mode transforms from dominantly ITG driven mode to dominantly ETG driven mode as the $\eta_{i}$ of the ions is reduced keeping $\eta_{e}$ fixed. The transition takes place at around $\eta_{i} \sim 1.6$. The corresponding real frequencies without (circles) and with (squares) the trapped electrons are shown in Fig. 13. Both reduce almost linearly with $\eta_{i}$, but because of the presence of the trapped electrons the later reduces faster than the former and tend to move in the electron diamagnetic direction. It is because of the fact that the mode inherent to the trapped electrons with finite $\eta_{e}$ starts to dominate over ITG driven mode as one reduces the $\eta_{i}$. 


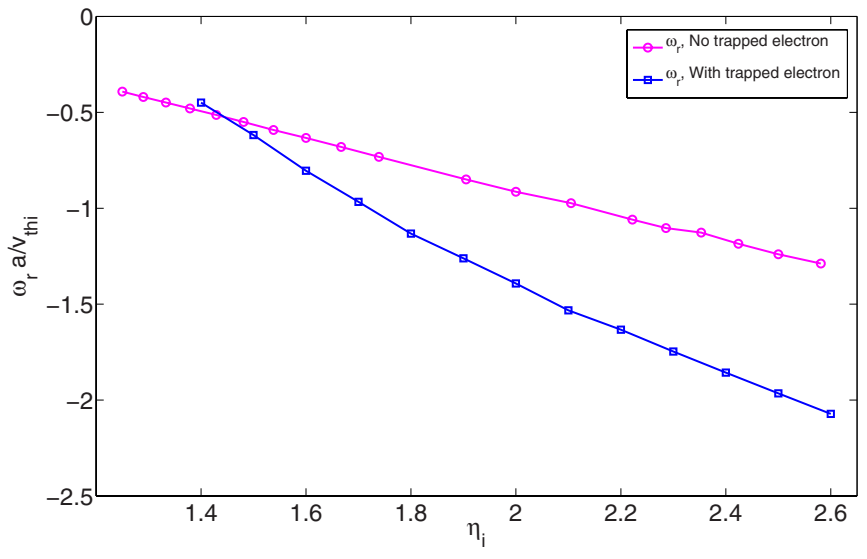

FIG. 13. (Color online) Normalized real frequency $\widetilde{\omega}_{r}$ vs $\eta_{i}$ scan for the SWITG mode at $k_{\theta} \rho_{L i} \approx 1.3$ with (solid line+square) and without (solid line+circles) the trapped electrons (from the global gyrokinetic model). $\eta_{e}\left(s_{0}\right)=2.5, q\left(s_{0}\right)=2.0, \hat{s}\left(s_{0}\right)=1.0, \tau=1.0$, and $\epsilon_{n}=0.1$.

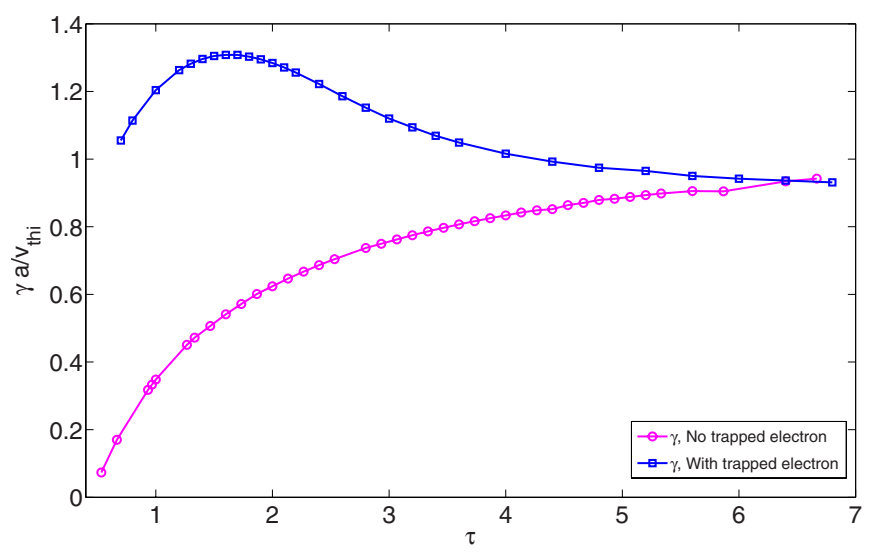

FIG. 14. (Color online) Normalized growth rates $\tilde{\gamma}$ vs $\tau$ scan for the SWITG mode at $k_{\theta} \rho_{L i} \approx 1.3$ with (solid line+square) and without (solid line +circles) the trapped electrons (from the global gyrokinetic model). $\eta_{e, i}\left(s_{0}\right)=2.5, q\left(s_{0}\right)=2.0, \hat{s}\left(s_{0}\right)=1.0$, and $\epsilon_{n}=0.1$.

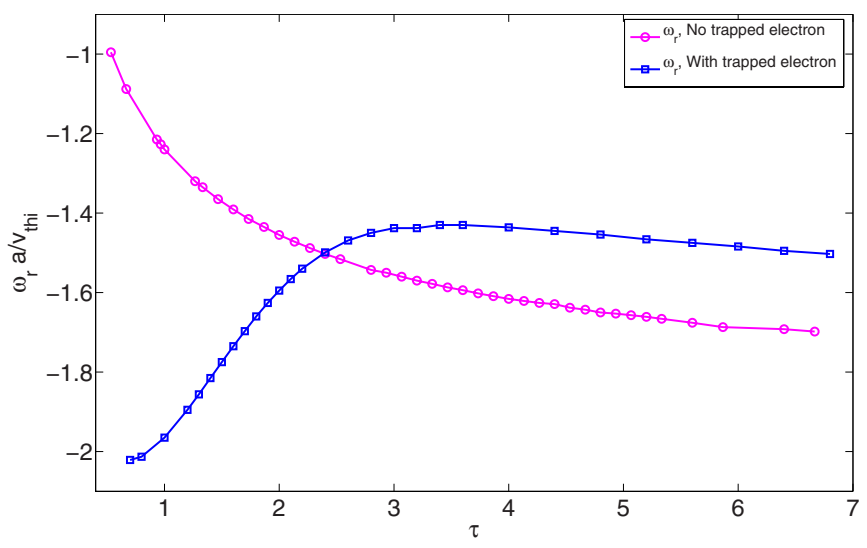

FIG. 15. (Color online) Normalized real frequency $\widetilde{\omega}_{r}$ vs $\tau$ scan for the SWITG mode at $k_{\theta} \rho_{L i} \approx 1.3$ with (solid line+square) and without (solid line+circles) the trapped electrons (from the global gyrokinetic model). $\eta_{e, i}\left(s_{0}\right)=2.5, q\left(s_{0}\right)=2.0, \hat{s}\left(s_{0}\right)=1.0$, and $\epsilon_{n}=0.1$.

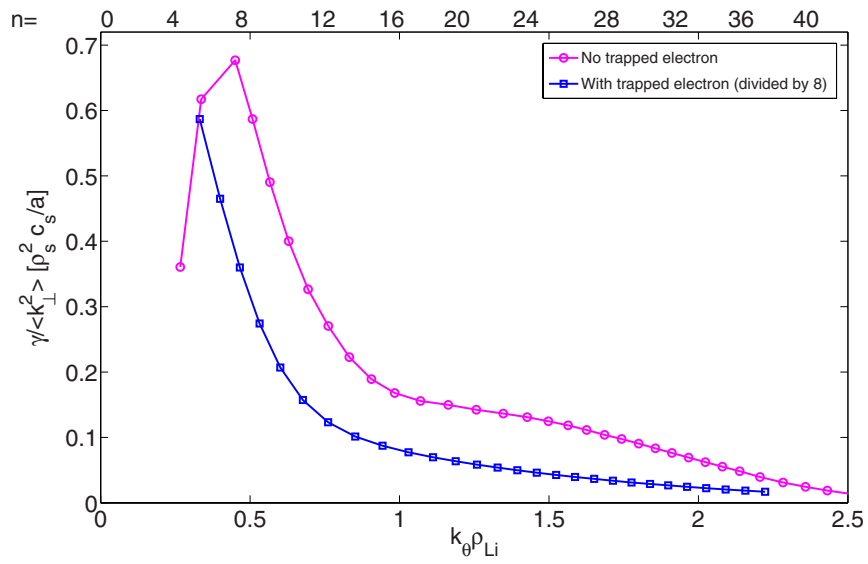

FIG. 16. (Color online) Mixing length estimate for transport coefficient $D_{\mathrm{ML}}=\gamma /\left\langle k_{\perp}^{2}\right\rangle$ of the ions in the ion gyro-Bohm units as a function of $k_{\theta} \rho_{L i}$; (a) the ITG and the SWITG mode without the trapped electrons (solid line+circle) and (b) the ITG and the SWITG mode with the trapped electrons (solid line+square) (from the global gyrokinetic model). Note that this curve is scaled down by a factor of 8 . The upper axis presents the corresponding toroidal mode numbers.

\section{D. $\tau$ scan}

To look at the temperature dependence of the mode, a $\tau=T_{e} / T_{i}$ scan is shown in Figs. 14 and 15 for the growth rate and the real frequency, respectively, using the global gyrokinetic model. The growth rate in Fig. 14 for the SWITG mode without the trapped electrons (circles) increases gradually with $\tau$, and at higher value it starts saturating. For the case with the trapped electrons (square), on the other hand, it increases initially and becomes maximum in the region $\tau$ $\approx 1-2$. It then falls and finally saturates. This can be explained from the fact that in the first case electrons are considered adiabatic, while the trapped electrons are included in the second case. So, at $\tau \gg 1, T_{e} \gg T_{i}$, the electron drive appears to be stronger reducing the growth rate in the later case. This is apparent from the Fig. 15 where the real frequencies are plotted against $\tau$. For the case with the trapped electrons, as $\tau$ increases the dominant electron drive pulls the real frequency toward the electron diamagnetic direction. Therefore, the real frequency decreases with increasing $\tau$ going toward more positive value, and then saturates. For the case without the trapped electrons, however, the real frequency rises initially with $\tau$ and then starts saturating in line with its corresponding growth rates.

\section{E. Mixing length estimation}

It will be interesting to calculate the heat diffusivity of the ions in the presence of the SWITG mode, over and above the conventional ITG mode. Within our linear model, we do this using the mixing length estimation where $\gamma /\left\langle k_{\perp}^{2}\right\rangle$, with $k_{\perp}=\sqrt{k_{r}^{2}+k_{\theta}^{2}}, k_{r}$ and $k_{\theta}$ being, respectively, the radial and poloidal wave numbers of the mode, is plotted with respect to the $k_{\theta} \rho_{L i}$. Thus, the heat diffusivity $\gamma /\left\langle k_{\perp}^{2}\right\rangle$ of the ions in the gyro-Bohm unit is depicted in Fig. 16 for the SWITG mode without (circle) and with (square) the trapped electrons. The diffusivity increases initially with $k_{\theta} \rho_{L i}$, peaks at $k_{\theta} \rho_{L i} \approx 0.5$, and then starts falling for the first case, but decreases mono- 
tonically for the second case. The maximum diffusivity shifts toward lower $k$ for both the cases with the trapped electrons and without the trapped electrons. It is to be noted that the values of the heat diffusivity for the case with the trapped electrons have been scaled down, dividing the actual values by 8 to show both the curves in the same scale. Thus, one can conclude that the trapped electrons rise the heat diffusivity substantially. One important point to be noted is that there is no peak specific to the $k_{\perp} \rho_{L i}$ of the SWITG mode, the whole spectrum of the heat diffusivity tends to peak at lower $k_{\perp} \rho_{L i} \leq 0.5$ despite the fact that the SWITG mode peaks at around $k_{\perp} \rho_{L i} \sim 1.5$.

\section{CONCLUSION}

In the present work, we have presented the features of the SWITG mode in the presence of the trapped electrons using a linear gyrokinetic model in the toroidal geometry, that treats both the species, namely, ions and electrons fully gyrokinetically, taking into account all the kinetic effects. Comparison of parametric dependencies for the two cases of the SWITG mode with and without the trapped electrons is presented. In line with the global model, we also compare the results from a local gyrokinetic model for the two cases, with and without the trapped electrons. This is for the first time where the SWITG mode is studied (1) in the presence of trapped electrons and (2) in the frame of a global gyrokinetic model. The major findings of the present work are the following.

- The trapped electrons have strong effect on the SWITG modes, rising the growth rate substantially. This is in contrast to the earlier conjecture that the trapped electrons may not be important for the SWITG mode.

- Although defined as SWITG, the two dimensional mode structure of the SWITG mode has been found to be quite global occupying a considerable fraction of the tokamak cross section for the chosen set of parameters. This establishes the necessity of a global model to study such a phenomenon.

- The most important observation is that, in the presence of the trapped electrons, the $L_{n} / R$ window for the existence of the SWITG mode gets widened. The toroidicity has strong stabilizing effect on the SWITG mode in the absence of the trapped electrons. Inclusion of the trapped electrons, however, has been found to make the mode stronger against the stabilizing effect of the toroidicity. Thus, the inference from this result is that the mode acquires toroidal-like nature in the presence of the trapped electrons in contrast to the slablike nature in the absence of the trapped electrons. The increased fraction of the trapped electrons with increased toroidicity is the main factor behind this flipping of the mode from the slab nature to the toroidal nature. The increased trapped fraction of the electrons with toroidicity reduces the adiabatic response of the electrons, which in turn enhances the formation of the space charge leading to a higher growth rate of the mode and hence the mode can withstand the effect of increased toroidicity.
- The SWITG mode is an ITG driven mode in the higher $k_{\perp} \rho_{L i}$ regime exhibiting a threshold in $\eta_{i}$. The mode persists even if the electrons are considered adiabatic. In the absence of the trapped electrons the mode vanishes below a critical $\eta_{i}$. But, in the presence of the trapped electrons, with the decreasing value of $\eta_{i}$, the mode does not vanish, rather it transforms itself from the dominantly ion mode to the dominantly trapped electron mode.

- The growth rate increases for lower values of $\tau$ but starts saturating at higher values of it. In the presence of the trapped electrons, the growth rate increases initially, but at higher values of $\tau$, where the electrons become hotter than the ions the growth rate falls and then saturates with the mode frequency tending to move toward the electron diamagnetic direction.

- An estimation of the ion transport based on the mixing length theory is done. The trapped electrons rise the heat diffusivity significantly. It is found that the ion heat diffusivity peaks at lower $k_{\perp} \rho_{L i}$. No significant diffusivity is observed at higher $k_{\theta} \rho_{L i}$ where the SWITG mode is strongest for both the cases with and without the trapped electrons.

The study of the trapped electron coupled SWITG mode including the effect of the equilibrium flow, electromagnetic perturbation with $B_{\|}$and/or $B_{\perp}$, Shafranov shift, and plasma shaping is an important area of research. This will be addressed in a future work.

\section{ACKNOWLEDGMENTS}

The authors (J.C. and R.G.) are thankful to A. Sen for careful reading of the manuscript. They are also thankful to IPR Computer Centre for its support during the course of this work. The computations were performed on a 34-node Xeon cluster with fast-ethernet interconnect at IPR. The CRPP authors were partly supported by the Swiss National Science Foundation.

${ }^{1}$ P. N. Guzdar, C. S. Liu, J. Q. Dong, and Y. C. Lee, Phys. Rev. Lett. 57, 2818 (1986).

${ }^{2}$ Y. C. Lee, J. Q. Dong, P. N. Guzdar, and C. S. Liu, Phys. Fluids 30, 1331 (1987).

${ }^{3}$ W. Horton, B. G. Hong, and W. M. Tang, Phys. Fluids 31, 2971 (1988).

${ }^{4}$ W. Dorland, F. Jenko, M. Kotchenreuther, and B. N. Roger, Phys. Rev. Lett. 85, 5579 (2000).

${ }^{5}$ R. Singh, P. K. Kaw, and J. Weiland, Nucl. Fusion 41, 1219 (2001).

${ }^{6}$ B. B. Kadomtsev and O. P. Pogutse, Nucl. Fusion 11, 67 (1971).

${ }^{7}$ M. N. Rosenbluth and M. L. Sloan, Phys. Fluids 14, 1725 (1971).

${ }^{8}$ B. Coppi and G. Rewoldt, Phys. Rev. Lett. 33, 1329 (1974).

${ }^{9}$ P. C. Liewer, Nucl. Fusion 25, 543 (1985).

${ }^{10}$ B. Coppi, M. N. Rosenbluth, and R. Z. Sagdeev, Phys. Fluids 10, 582 (1967).

${ }^{11}$ F. Romanelli, Phys. Fluids B 1, 1018 (1989).

${ }^{12}$ W. Horton, Rev. Mod. Phys. 71, 735 (1999).

${ }^{13}$ A. I. Smolyakov, M. Yagi, and Y. Kishimoto, Phys. Rev. Lett. 89, 125005 (2002).

${ }^{14}$ A. Hirose, M. Elia, A. I. Smolyakov, and M. Yagi, Phys. Plasmas 9, 1659 (2002).

${ }^{15}$ Z. Gao, H. Sanuki, K. Itoh, and J. Q. Dong, Phys. Plasmas 10, 2831 (2003).

${ }^{16}$ Z. Gao, H. Sanuki, K. Itoh, and J. Q. Dong, Phys. Plasmas 12, 022502 (2005).

${ }^{17}$ Z. Gao, J. Q. Dong, and H. Sanuki, Phys. Plasmas 11, 3053 (2004). 
${ }^{18}$ Z. Gao, H. Sanuki, K. Itoh, and J. Q. Dong, J. Plasma Phys. 72, 1249 (2006).

${ }^{19}$ Y.-K. Pu and S. Migliuolo, Phys. Fluids 28, 1722 (1985).

${ }^{20}$ S. Brunner, M. Fivaz, T. M. Tran, and J. Vaclavik, Phys. Plasmas 5, 3929 (1998).

${ }^{21}$ G. L. Falchetto, J. Vaclavik, and L. Villard, Phys. Plasmas 10, 1424 (2003).

${ }^{22}$ R. Ganesh, P. Angelino, J. Vaclavik, and L. Villard, Phys. Plasmas 11, 3106 (2004).
${ }^{23}$ P. Angelino, Ph.D. thesis, EPFL, 2006.

${ }^{24}$ R. Ganesh and J. Vaclavik, Phys. Rev. Lett. 94, 145002 (2005).

${ }^{25}$ S. Brunner, Ph.D. thesis, EPFL, 1997.

${ }^{26}$ J. Chowdhury, R. Ganesh, P. Angelino, J. Vaclavik, L. Villard, and S. Brunner, Phys. Plasmas 15, 072117 (2008).

${ }^{27}$ J. Chowdhury, R. Ganesh, S. Brunner, J. Vaclavik, L. Villard, and P. Angelino, Phys. Plasmas 16, 052507 (2009).

${ }^{28}$ K. L. Wong, N. L. Bertz, T. S. Hahm, and E. Synakowski, Phys. Lett. A 236, 339 (1997). 